\title{
ESTUDIO COMPARATIVO DEL RENDIMIENTO ACADÉMICO EN LA ASIGNATURA DE MATEMÁTICA ENTRE ESTUDIANTES DE EDUCACIÓN PRIMARIA QUE PARTICIPAN DEL PROGRAMA INFORMÁTICA EDUCATIVA Y LOS QUE NO PARTICIPAN, EN LA DIRECCION REGIONAL DE EDUCACIÓN DE SAN JOSÉ
}

Sergio Calvo Vargas

\section{Introducción}

El Ministerio de Educación Pública, en la Girima década. ha impulsado una serie de políticas educativas apoyadas generalmente en prorectos educativos en cooperación con entidades internacionales y gohiernos extranjeros, a saber: Banco Mundial. Banco Interamericano de Desarrollo. Organización Internacional para el Trabajo. LNESCo, gobiemos de Holanda. Suiza $\mathrm{y}$ otros.

El propósito sustantivo de usas colaboriaciones es mejorar las condiciones del ambiente formativo del educando y las condiciones laborales del eciucador. orientadas a fortalecer y enriquecer la calidad de la educación costarricense desde los primeros niveles educativos.

Como respuesta a la necesidad de contribuir al mejoramiento de la calidad de la educación costarricense y de democratizar el sistema educativo, tanto en término cualitativos como de acceso a los adelantos de la ciencia y la tecnología, se creó el "Programa de Informática Educativa MEP-FOD" (llamado en adelante P.I.E.).

Las investigaciones en este sentido se han dirigido, en algunos casos, a evaluar el impacto del P.I.E. en el sistema escolar, con la intención de obtener resultados sobre los alcances de los objetivos propuestos por el P.I.E., principalmente mediante enfoques cualitativos.

Aunque es relevante obtener resultados cualitativos del programa, también es de suma importancia dilucidar los alcances cuantitativos que han logrado los educando que están participando en el proceso educativo planificado $y$ ejecutado por el P.I.E., en lo que respecta al reforzamiento que ha tenido el Programa en las materias básicas, como son: Matemática, Ciencias. Español y Estudios Sociales.

También comviene constatar dichos alcances cuantitativos por ser el P.I.E. un proyecto innovador, donde se vitizan gran cantidad de recursos humanos $y$ financieros con el fin de reforzar el aprendizaie de los educandos.

Este estudio se propone investigar el aprovechamiento unntatito que han desarollado los estudantes de quinto grado de Educación Primar. uhicados en la Región Educatid a sur lose especificamente en la asignatura de Matsmitica después de haber recuhido una insmación de reforzamiento medante el Si-tema Computarizado Logo Writer. Y comparar 'os renlados ohtenidos con otros catudianter de mismo nivel que no recibieron dicho aprendiazie

\section{Problema}

- Existe diferencia significativa en el rendimiento académico en la asignatura de Matemática entre estudiantes de quinto grado de escuelas públicas de la Región Educativa de San José, que han recibido el Programa de Informática Educativa, y los que no han participado en dicho Programa?

\section{Hipótesis de investigación}

Sí existe diferencia en el rendimiento académico en la asignatura de Matemática, entre estudiantes de quinto grado de escuelas 
públicas de la Región Educativa de San José, que han participado del Sistema Computarizado Logo Writer, con respecto a otros estudiantes que no han participado.

\section{Definición de variables}

Las variables que orientan el desarrollo de la presente investigación, se definen de la siguiente manera:

Variable dependiente: Rendimiento académico.

Definición conceptual: El logro obtenido por los estudiantes con respecto a los objetivos y contenidos del I y II trimestre, del Programa le Estudio oficial de quinto grado, en la aisignatura de Matemática.

Definición operacional: Está constituida por el puntaje obtenido por los estudiantes en la prueba de Matemática, que mide los contenidos básicos del I y II trimestre del Programa de Estudio de quinto grado, mediante una muestra representativa.

Variable independiente atributiva: Sistema Computarizado Logo Writer.

Definición conceptual: Aplicación del Lenguaje Logo Writer, el cual permite utilizar la computadora como herramienta que facilita el desarrollo de habilidades cognoscitivas en la asignatura de matemática.

Definición operacional: Está constituida por la agrupación que para efectos de esta investigación se hizo de las instituciones de la muestra representativa de la Región Educativa de San José.

\section{Niveles de la variable:}

Instituciones donde se imparte el Lenguaje Logo Writer del P.I.E.

Instituciones donde no se imparte el Lenguaje Logo Writer del P.I.E.

\section{Limitaciones}

Por ser un diseño ex-post facto, no permite controlar las terceras variables que influyen en los resultados de la investigación.

- La investigación no cubre toda la población del país, solamente la Región Educativa de San José, limitando la generalización de los resultados.
El diseño no se ubica dentro de un modelo de investigación causa-efecto, debido a la no manipulación de la variable independiente (Sistema Logo Writer).

El estudio comparativo se ubica únicamente en el nivel de quinto grado, por lo que no se puede relacionar los resultados de este estudio con otros grados de educación primaria, ni tamaño de escuela.

\section{Marco teórico}

\section{Concepto de aprendizaje}

Diferentes autores han dado su criterio y explorado sobre el aprendizaje y sus implicaciones en $e l$ desarrollo educativo, donde, a través suyo, el hombre podría ser definido como un ser que aprende continuamente, ya que su vida transcurre cambiando el comportamiento descie que nace hasta que muere.

Por lo tanto:

"El hombre no solo se ha mostadie cieseoso de aprender sino que con frecuencia su curiosidad le ha impedido tratar de averiguar como aprende". (Bigge, 1988, p. 17).

Añade el autor:

"dentro de la teoría del campo cognoscitivo, el aprendizaje, ... es un proceso de interacción en el cual una persona obtiene nuevas estructuras cognoscitivas o insights, o cambia las antiguos". (Bigge, 1988, p. 236).

Uno de los problemas más serios y comunes que se encuentran en las escuelas radica en lograr articular los recursos en forma efectiva al aprendizaje escolar, provocando ineficiencia en el aprendizaje. Por ello diferentes profesionales de diferentes áreas de especialización llegaron a plantearse la necesidad de estudiar si las escuelas estaban estimulando o no el aprendizaje (Pereira, 1991, p. 26). Por ello, es importante que los educandos se involucren en procesos educativos en los cuales puedan adquirir nuevas formas de comportamiento y conocimiento, o que puedan modificar formas anteriores.

Podría afirmarse que detrás de toda práctica escolar, hay presente un concepto de aprendizaje que guia la labor educativa y establece de igual manera los roles por seguir 
para los participantes en el proceso; este es el caso del Programa de Informática Educativa (P.I.E.), el cual basa su sustento epistemológico en la teoría piagetiana, cuyo concepto de aprendizaje comprende la construcción del conocimiento, no sólo desde un punto de vista funcional explicado por los procesos de asimilación y acomodación, sino también, descle la perspectiva estructural.

Para comprender la totalidad de la relación sujeto-objeto, otros autores afirman:

\footnotetext{
"No hay que olvidar que el individuo solamente aprende wando se enfrenta con situaciones problemáticas, para ... uales no tengan respuestas adecuadas de comporta...ent: induciendolo a buscarlas y descubrirlas". Seric1, 1973, p. 214).
}

Segin la praxis de la F.O.D., el accionar -.endizaje del niño en el laboratorio se da:

in proceso. desde las primeras sesiones que son :1 is actividades de exploración, hasta etapas pos-2.:es en que los niños aprenden los fundamentos de la aramación en Logo, no como un objetivo en si, sino n. una herramienta ... que deben ejercitar su iniciativa. and a capaciad de planear y resolver proble entra del contexto de un tema escolar determinado". $\equiv-2.1001,0.42$.

De ahi la importancia de una convenien$\because$ - Esertación para un trabajo didáctico, pues $\therefore$ EEhen atender no solamente las necesida$\therefore$ E: fropias de la edad del niño, sino también - cacidad organizativa que posee y la posiS.ifad de desarrollar un intercambio activo ca: os objetos y situaciones diarias de aprendizaje. que para él son las que cobran sentido. En este aspecto, el aprendizaje es un proceso mulidimensional, intersubjetivo y significativo para ios sujetos, pues son ellos lo que construyen $y$ reconstruyen su propio conocimiento (García. I., 1995, p. 5).

\section{Cobertura del Programa de Informática Educativa (P.I.E.)}

En respuesta a inquietudes de la sociedad costarricense en torno a la calidad de la educación pública, se inicia el P.I.E. en un esfuerzo conjunto del Ministerio de Educación Pública y la Fundación Omar Dengo (llámese en adelante FOD) , entidad que ha proporcionado el aporo logístico requerido para su puesta en marcha, funcionamiento adecuado y crecimiento cualitativo. (Fonseca, 1991, p. 46). Cada laboratorio del P.I.E. está integrado por 20 computadoras IBM y una impresora, uhicados principalmente en escuelas rurales y urbanas marginales del país. Actualmente, el P.I.E. cuenta con 159 laboratorios $(3.000 \mathrm{com}-$ putadoras) y 352 maestros entrenados, que atienden un total de 165.293 niños y niñas, que representan el $30 \%$ de la población escolar del país (estadísticas generales del P.I.E., junio de 1993).

Los laboratorios son atendidos por docentes especialmente capacitados para ello (tutores) y por docentes responsables de cada grupo. El ambiente de aprendizaje que se trata de generar en estos laboratorios se fundamenta en la concepción de la computadora como herramienta de aprendizaje, y en la utilización del lenguaje Logo, considerado teóricamente como software educativo capaz de permitir al usuario hacer uso de un procesador de texto y de un graficador, creado por el Dr. Seymour Papert y Marvin Minsky en el Laboratorio de Inteligencia Artificial del Instituto Massachusetts (M.I.T.) en 1968, para la construcción del conocimiento de los niños, hasada en la teoría del aprendizaje de Jean Piaget (González, 1994, p. 7).

La base epistemológica en que se desenvuelve el P.I.E se susienta en al construccionismo escrito por Aaron Falhel que nos dice:

"La teoría del constucionismo atima que el aprendizaje es mucho mejor cuanditis nnos se comprometen a la construcción de un prisuce significative, tal como un castillo de arena, un prema. una máutiza.. El construccionismo involucra dos tipos de constricérn cuando los niños construyen cosas en el mundo externo. simultáneamente construyen conocimiento al interur de sus mentes. Este nuevo conocimiento les permite entonces construir cosas mucho más sofisticadas en el mundo externo. lo que genera más conocimiento. y así cuceriamente en un ciclo autorreforzante... Elementos de fantasía. imaginación y creatividad contribuyen con la calidad $v$ ia unicidad del producto final que ostenta el toque personal del creador..." (Falbel, 1993, p. 3).

Logo asume dos funciones importantes como son, por un lado un lenguaje de programación y por otro lado, es un ambiente educativo. La versión que actualmente se utiliza en Costa Rica es Logo Escritor (Logo Writer), programa que permite la utilización conjunta 
de gráficos y textos, según nos describe el Dr. Papert:

"Logo es un lenguaje de programación proyectado par" ser accesible a los niños en cuanto retienen smitianted mente el poder simbólico de otros lenguates amputact. nales (Papert, 1980). Logo es el nombre de und hile de la educación dentro de una creciente familáa de ion guajes de computación que la acomparia. Los rasgos cat racteristicos de la familia de los lenguajes ingug incluyey

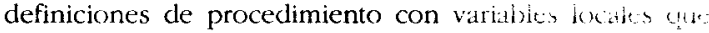
permiten la recursión, de tal modo, en Lry es protit definir nuevas órdenes y funciones que luteo puederi usarse texactamente como las primitivas. Lngo es un ien guaje interpretativo. Esto significa que puede utilizarse en forma interactiva. Los modernos sistemas de Logro pueden operar con listas cuyos componentes pueden ser ellos mismo listas listas de listas. (.) Debe recordarse que i.sIs no ha sidu concebido como un producto final ni se $k$ of rece como "el lenguaje definitivo"... Algo mejor es posible Algunas versiones tienen más recursos". (Paper. $1987,[0,240-2,7)$

Cabe indicat que se menciona como Geriento de apriyo. el uso de Logo en otros pares cono thincia. inglatera y E.UA (Fonseca. $1908 . p=5$ pero no se especifica si se imparte coms unica opcian para el uso del computador en li zidiela cono ocurre actualmente en Costa Rica.

Facilitadores del proceso de aprendizaje desde la perspectiva del P.I.E,

El P.I.E. para la enseñanza primaria pública se inició el 20 de diciembre de 1988 , siendo el primer programa puesto en ejecución conjuntamente por el MEP y la Fundación Omar Dengo.

El P.I.E. tiene su fundamento en el uso de la computadora para incentivar el desarrollo de procesos cognoscitivos al tiempo que busca enriquecer el currículum escolar creando un ámbito de exploración y construcción del conocimiento por medio del desarrollo de proyectos vinculados a los programas oficiales de estudio, para alcanzar este propósito. "E] maestro puede escoger la ruta del idioma o la ruta de las gráficas y la matemática para introducir a los niños al mundo de la computadora y su uso educativo" (Fonseca, 1991, p. 42).

Los niños del I Ciclo asisten regularmente dos veces por semana, en sesiones de 40 minutos cada una, mientras que los del II Ciclo utilizan dos lecciones seguidas para un total de 80 minutos. En cada microcomputadora trabajan simultáneamente dos niños o niñas.

En el laboratorio del P.I.E., el tutor interisctúa con el alumno de manera que facilite sensitiva y creativamente el proceso de aprenrizacic dejando atrás los modelos tradicionales estructuracios $v$ rigidos. De manera similar, el matsro de grado deberá realizar un cambio protindo en su actitud, tanto en relación con los contenidos como con la dinámica de trabajo on auh. (González, 1994. p. 13).

Según Fonseca y Schaffer (1990):

"Es precinci que el docente aprenda a verse a sí mismo como un profesional on una época de imansición, que debe atuat fo pucnte entre sa alummos y las innovaciones anologita y echativas". (1900. p. 5)

De esta manera. el maestro(a) de grado, junto con el tutor, utilizan para la construcción del conocimiento de los alumnos, la filosofía social y educativa que está sustentada y apoyada en los objetivos diseñados por el P.I.E.

\section{Objetivos}

1. Contribuir al mejoramiento de la calidad de la educación primaria en todos sus aspectos.

2. Contribuir al desarrollo de una nueva actitud de niños y maestros ante la ciencia y la tecnología.

3. Familiarizar a los estudiantes y a los educadores con el uso de la computadora y algunas de sus aplicaciones.

4. Estimular procesos de aprendizaje, creatividad y pensamiento lógico en los estudiantes y los docentes.

5. Complementar la enseñanza-aprendizaje de diversas disciplinas, especialmente aquéllas que conduzcan al desarrollo del pensamiento lógico.

6. Contribuir al mejoramiento de la calidad de la enseñanza de las materias básicas: Matemática, Ciencias, Español y Estudios Sociales. 


\section{Rendimiento académico}

En el sistema educativo costarricense, en lo que respecta al nivel de logro del alumno, se rige de acuerdo al marco filosófico establecido por la Pólítica Educativa Hacia el Siglo XXI, aprobada por el Consejo Superior de Educación, el 8 de noviembre de 1994. La misma sustenta al "Marco de Referencias y Directrices Técnicas para la Evaluación en el Sistema de Educación Formal".

Esta relación entre el proceso y el producto se encuentra mediada por la acción de los maestros(as) y de los profesores(as).

El rendimiento académico en las escuelas $\because$ colegios oficiales se rige mediante el "Marco de eferencia técnico y normas en materia de craliaición de los aprendizajes y el desarrollo -..i-ifectro y ético de los estudiantes", cuyas A.m. Lei aimno(a) con el propósito de expresar la - viaridad obtenida, en un trimestre, semestre, en el año, según el nivel en que se encuentre.

Es común encontrar que el rendimiento aradémico es definido en las escuelas y colegios oficiales mediante pruebas escritas. Por lo tanto:

e-i de rendimiento académico tienen como fin valorar Li: encia de un curso de formación específica. De hecho. cuziguer examen tipificado realizado en un curso escolar representa un test de rendimiento". (Azofeifa, 1978, p. 21).

Por otro lado Kerlinger (1975), hace referencia a que en el rendimiento académico intervienen, entre otros, factores tales como las características del alumno en cuanto a inteligencia, actitudes, hábitos, gustos, carácter del profesor, metodologia, formación profesional. También incluye la organización del sistema educativo, el ambiente familiar $y$ los factores sociales.

En la actualidad, nadie duda de la influencia de estos factores en el rendimiento académico de la Matemática en una sociedad tecnológica, en la vida cotidiana y en la formación integral del educando. Por eso, el rendimiento académico en esta asignatura nos revela que tan exitoso o fracasado se puede presentar el proceso de aprendizaje en el alumno(a).

Algunos autores opinan que:

'E gran fracaso en el aprendizaje de la Matemática debe arribuirse. fundamentalmente. a la metodología que utiliza un número considerable de profesores, pues no propician en sus alumnos el desarrollo de la capacidad lógica del pensamiento". (Pardo, 1990, p. 12).

\section{Otros estudios revelan que:}

"El desarrollo de las nociones lógico-matemático en los ninos de la muectra estudiada, no está influenciada por la zuna ruá o urbana... ni por la asistencia". (Blanco y otus 1981. p bis.

Esto es importante. va que pone en evidencia el hecho de que, desde el inicio, el proceso educativo del educando evidencia un descuido en la formación de conceptos matemáticos, atribuidos en muchos casos a las metodologías aplicadas a otro tipo de variables.

Para mejorar el proceso de aprendizaje, Papert, S., citado por Dobles, C. (1994), señala que la computadora colabora con el desarrollo de los procesos cognoscitivos del niño, por un lado como instrumento, y por otro lado, como posibilitador de la comprensión conceptual de nociones abstractas, como de la sintaxis de la lengua o de la Matemática.

El logro de un buen rendimiento académico es el reflejo de un aprendizaje de calidad con enseñanza de calidad facilitada principalmente por el docente. Para ello es necesario articular en forma eficiente los niveles cognoscitivo, afectivo y motor del niño, donde, a través de los recursos disponibles, pueda desarrollar a plenitud la lógica matemática con mayor acierto.

Investigaciones sobre logros del P.I.E. en la Educación Primaria

El P.I.E. desde su origen. ha sido objeto de múltiples evaluaciones. Dentro de las últimas cabe citar la realizada por la Dra. Zayra Méndez (1988-1992). titulada "Evaluación del Programa de Informática Educativa de I y II Ciclo". El propósito fue obtener un diagnóstico de la condición inicial y razonamiento lógico, matemático espacial y en creatividad, de una muestra de niños que asistian a escuelas con o sin laboratorio de informática. Para la ejecución de este se utilizó como punto de partida un pre-test. Se obtuvo como resultado que no existian diferencias significativas entre ambos grupos de niños. (Vargas, Salas y Víquez, 1993. pp. 88-89). 
En otro estudio se señala que en la investigación nombrada anteriormente no se realizó el pos-test de la forma en que prefiguró en la primera etapa de la evaluación, por lo que puede haber afectado sensiblemente los resultados de la misma. (Zúñiga, García y Murillo, 1992. p. 2).

Otras investigaciones en relación con el desarrollo del P.I.E. son las realizadas dentro del área de Investigación de la FOD, orientadas principalmente a evaluar los alcances del P.I.E. en el ambiente Logo, a la luz de un enfoque metodológico cualitativo.

"El papel de las interacciones ante un objeto nuevo" (Anny González. 1994).

"Significaciones en el contexto escolar y actividad cognitiva". (Cecilia Dobles y Magali Zúñiga, 1994).

"Impicaciones de los paradigmas en las conceptualizacio nes pedagógicas". (Jacqueliné García, 1995).

"Evaluación macrounalitica de contexto solpre una mucstra de escuelas que amende 1988-1993". (Cecilia Dobles y Jacqueline Gurciat. 190;

"Evaluacion formativa. l’rograma de Informática Educativa MEP-FOD". (Dobles C. Y (itros. 1995)

En la última investigación realizada en 1995 entre sus múltiples conclusiones se obtuvo que:

"Los procesos cognitivos observados en e! trabajo de niños y niñas con la computadora no llegan. en la mayoría de los casos a alcanzar el nivel esperado (alto nivel), análisis, anticipación, metocognnición, abstrucción, equilibrio. etc. (Dobles, C. y otros. 1995. p. 19).

\section{Metodología}

\section{Tipo de estudio}

Este estudio es de tipo ex-post facto. dentro del ámbito de la investigación educativa. Señala Kerlinger (1982) que la expresión ex-post facto significa "a partir de lo que ya ha acontecido" (pág. 192) y denota algo que sucede o se realiza después de un fenómeno que tiene efectos retroactivos sobre el acontecimiento; en este caso, después de haber recibido dos trimestres de Lenguaje Logo Writer.

\section{Población}

Los sujetos de estudio son 3.384 estudiantes de quinto grado de las escuelas públicas de la Región Educativa de San José.

\section{Muestra}

Las escuelas se seleccionaron por medio del inuestreo aleatorio simple que a continuación se detalla.

Se eligieron los quintos grados, debido a que el P.I.E. cubre ese nivel en todas las escuelas, no así en otros niveles. Por tal motivo, se tomaron tamaños de escuelas 3,4 y 5 , desechándose las 1 y 2 , ya que éstas carecen del P.I.E.

El total de escuelas de tamaño 3,4 y 5 en la Región Educativa de San José, 85 no tienen P.I.E. y 33 tienen P.I.E., de las cuales se eligió una muestra aleatoria del 20\%; es decir, 17 escuelas sin P.I.E. y 8 escuelas con P.I.E. (ver cuadro No. 1).

De los niños y las niñas que reciben lecciones en las escuelas seleccionadas, se elegirán como muestra aleatoria el $20 \%$ de estudiantes por escuela para participar en la prueha, para un total de 678 alumnos.

En el cuadro № 1 aparecen las escuelas seleccionadas para la muestra.

\section{Instrumento}

La prueba está confeccionada para aplicarse como pos-test a estudiantes de quinto grado de escuelas oficiales. Se hizo con el fin de medir el logro de los contenidos en la asignatura de Matemática y determinar, de acuerdo con los resultados, las diferencias entre estudiantes que han recibido el Programa de Informática Educativa en relación con estudiantes que no han recibido dicho programa.

La prueba fue construida bajo la modalidad de prueba con referencia a normas. Para tal fin, se siguieron los siguientes pasos metodológicos:

Se revisó el programa de quinto grado de Matemática y se construyeron objetivos que comprendian contenidos matemáticos básicos del I y II trimestre del programa.

Se sacó un listado de diez objetivos, y se confeccionaron de dos a cuatro ítemes por ca- 
Cuadro No. 1

Cantidad de estudiantes por tamano de escuela pública de la Dirección Regional de San José, que participan en lat muestra

\begin{tabular}{|c|c|c|c|c|c|c|c|}
\hline \multicolumn{4}{|c|}{ Tamaño } & \multicolumn{4}{|c|}{ Tamaño } \\
\hline Escuelas c/P.I.E. & 3 & 4 & 5 & Escuelas s/P.I.E. & 3 & 4 & 5 \\
\hline San Luis de Acosta & 5 & & & Esquivel Morales (Pieclades) & 18 & & \\
\hline La Peregrina (Uruca) & 20 & & & & & & \\
\hline Rep. Argent. (Merced) & & 26 & & Franklin Roosevelt (San l'edro) & 26 & & \\
\hline Omar Dengo (Hospital) & & & 30 & & & & \\
\hline Rincón Gde. (Pavas) & & & 49 & Las Mercedes Aserri! & $1 \overline{3}$ & & \\
\hline Central S. Sebastián & & & 44 & & & & \\
\hline Rep. Venez. (Escazú) & & & 36 & Gabriel Brenes (Awrri) & 15 & & \\
\hline Abraham Lincoln & & & 53 & & & & \\
\hline (Alajuelita) & & & & Dos Cercas (Devanparaclos) & 12 & & \\
\hline & & & & Ciuclaclela (1'avas) & 25 & & \\
\hline & & & & Quince de Setiembre (Hatillo) & 10 & & \\
\hline & & & & San Felipe de Alajuelita & & 4 & \\
\hline & & & & Juan Pestolozzi (Purral) & & 27 & \\
\hline & & & & José Cubero Muñoz (Mata de l'litano) & & 20 & \\
\hline & & & & Sinta Marta (San Fco. dos Ríos) & & 20 & \\
\hline & & & & República de Perá (El Carmen) & & & 36 \\
\hline & & & & Vitalia Madrigal (El Carmen) & & & 39 \\
\hline & & & & Naciones Uniclas (Catedral) & & & 29 \\
\hline & & & & Carlos Sanabria (Patvas) & & & 47 \\
\hline & & & & Mantel Jelgrano (Hatillo) & & & 30 \\
\hline & & & & Pilar Jinenter (Guadalupe) & & & 42 \\
\hline subxotal P.I.E. & 25 & 26 & 212 & $\triangle A+A d \times O P E$ & 121 & 71 & 223 \\
\hline
\end{tabular}

da objetivo, según los resultados del cuadro de balanceo. Para la redacción de cada ítem. se tomaron como referencia los constructos, variables e indicadores derivados de los objetivos.

La prueba se estructura de la siguiente manera:

Aspectos generales para la administración de la prueba.

Portada.

Instrucciones para el estudiante.

Selección única.

Desarrollo.

- Hoja de respuesta.

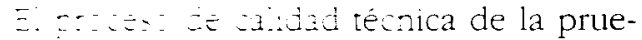

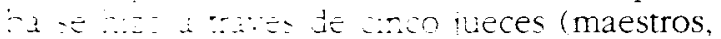

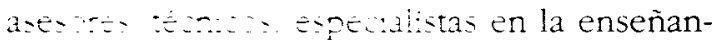

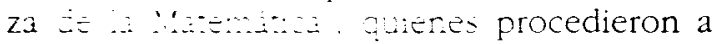
otrece: a la redana is aguros itemes. Para ello se les fachito a pricia original, con sus respectivas instruczicne: jurio, 1995).

Para determinar la consistencia interna del instrumento se aplicó la prueba a un grupo de 30 estudiantes de una escuela oficial (agosto, 1995). Luego se utilizó el coeficiente Alfa de Cronbach: este procedimiento se realizó mediante la aplicación del paquete estadís- 
tico SPSS, y se obtuvo un Alfa de 0,82 , lo que indica que el grado de confiabilidad del instrumento es fuerte y aceptable para el tipo de prueba.

Finalizado el análisis de ítemes e índices de discriminación y sustitución de ítemes, se estructuró una nueva prueba que contenía 30 ítemes de selección única y tres problemas en la sección de desarrollo (octubre, 1995).

\section{Análisis de resultados}

El presente apartado expone los resultados que corresponden a las relaciones planteadas en la hipótesis de investigación de este estudio, divididos en dos secciones:

Datos descriptivos.

Análisis inferencial.

Datos descriptivos

Los resultados obtenidos per tamis de escuela y grupo total. se presentir s continisción:

\section{Cuadro $\mathrm{S}$ :}

Número de casos. mediats aritméticit : a-d te te terersión para las dos muestras independiezes anan de excuela y en total (MEP - 1, 3,5

\begin{tabular}{lrcccc}
\hline $\begin{array}{l}\text { Tamaño } \\
\text { escuela }\end{array}$ & Grupo & $\begin{array}{l}\text { Número } \\
\text { calsos }\end{array}$ & $\begin{array}{l}\text { Media } \\
\text { Arit. X }\end{array}$ & $\begin{array}{l}\text { Desialc } \\
\text { stánd. s }\end{array}$ & $\begin{array}{l}\text { Varianza } \\
\text { s }\end{array}$ \\
\hline T-3 & NO PIE & 121 & 15,27 & 5,71 & 27,70 \\
& & 25 & 14,76 & 2,95 & 8,70 \\
T-4 & NO PIE & 71 & 15,79 & 5,47 & 29,92 \\
& PIE & 26 & 15,07 & 4,77 & 22,75 \\
T-5 & NO PIE & 223 & 16,99 & 5.52 & 30,47 \\
\multirow{2}{*}{ Total } & PIE & 212 & 15,34 & 5,12 & 26,21 \\
& NO PIE & 410 & 16,43 & 5,58 & 31,13 \\
& PIE & 268 & 15,29 & 4,88 & 23,81 \\
\hline
\end{tabular}

Se observa en el Cuadro № 2 que el número de casos NO P.I.E. es mayor que el de P.I.E., para cada tamaño de escuela. Se explica por el diseño de 'muestra utilizado en este estudio.

En relación a las medias aritméticas se observa que las correspondientes al grupo NO P.I.E., son mayores que las del grupo P.I.E., y esto se nota para cada tamaño de escuela $y$ para el grupo total. Estos resultados indican un rendimiento más alto en la prueba de $\mathrm{Ma}$ temática en los estudiantes del grupo NO P.I.E., que en los del grupo P.I.E..

Sin embargo, por tamaños de escuela, se observa que las T-3 presentan las medias aritméticas más bajas en ambos grupos (NO P.I.E.: 15,27 y P.I.E.: 14,76), mientras que las escuelas $T-5$ muestran las medias aritméticas más altas (NO P.I.E.: 16,99 y P.I.E.: 15,34). Estas diferencias en las medias aritméticas indican que los estudiantes de escuelas T-5 tienen notas mejores que los de escuelas T-3, aunque no nos ofrece infomación que determine si esa diferencia se debe a puntajes extremos de los estudiantes de ambos grupos en la curva normal

Con respecto a las medias aritméticas totales, la del grupo \O P.I.E. (X: 16,43), es mayor a la del grupo P.I.E. (X: 15.29) lo que significa ulue exisen diferencias entre estos dos grupo an un aero rédmento académico

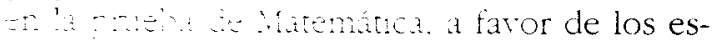
ande

Ea $\therefore$ U. $N=2$ se nota que conforme nure el tamaño de la escuela, mayor $\because$ a serración estándar, lo que se presenta en ambos grupos (P.I.E. y NO P.I.E.). Estos resultados indican que los grupos en las escuelas de tamaño 5 poseen puntajes más dispersos que estudiantes de escuelas de tamaño 3 y 4 . Por el contrario, las escuelas de tamaño $3(s=2,95$ y 5,71$)$, poseen puntajes menos dispersos con respecto a la media aritmética.

Por otro lado, la desviación estándar indica la homogeneidad relativa de los dos grupos (P.I.E. y NO P.I.E.) en Matemática. Por lo tanto, existen mayores diferencias individuales entre los estudiantes del grupo NO P.I.E. que los del grupo P.I.E., debido a que las desviaciones estándar de los primeros son mayores en los tres tamaños de escuela: T-3 $(5,71)$, T-4 $(5,47)$ y $\mathrm{T}-5(5,52)$.

Al comparar las desviaciones estándar de los grupos según el tamaño de escuela, se desprende del Cuadro № 2 que los grupos de la escuela de tamaño 3 presentan desviaciones estándar muy diferentes (P.I.E.: 2,95, y NO P.I.E.: 5,71), manifestándose mayores diferencias individuales de los estudiantes en este tamaño de escuela que en las más grandes (T-4 y T-5). 
Además, se puede observar una mayor desviación o dispersión de los puntajes con respecto a la media aritmética en el grupo NO P.I.E. $(S=5,58)$, tendenciá qut e rantuvo en los grupos de las muestra pur lat. de escuela.

Una regla práctica para estmual id lesiación estándar y evitar errores, consiste en a . . la razón del rango a la desviación estincia: muy raras veces es menor que 20 major 6 (Runyon y Haber, 1984). En particular a (....e estudio, el rango se establece entre $2,95-5.58$. lo que indica que no se están cometiendo errores de predicción con los datos obtenidos en este estudio.

Por otro lado, los grupos que presentan las varianzas más altas son los cle tamaño 5 , ando evidencia que existen diferencias de ancalos ten ambos grupos, que están afectan1. L homogeneidad de la varianza, revelando a existencia de puntajes extremos en las dos colas de la distribución.

Aunque la falta de homogeneidad de las varianzas puede afectar las interpretaciones del estudio, es necesario determinar qué tan ignificativas son cotas diferencias en cada tamano de escuela y grupos totales en muestras mitendientes
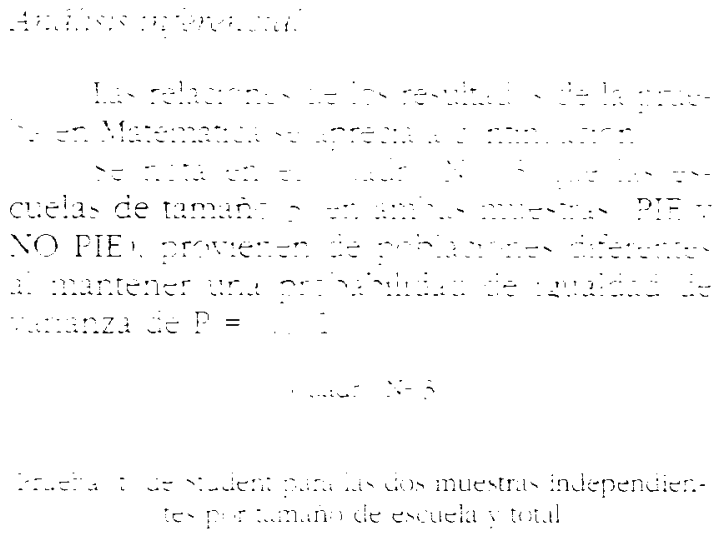

\begin{tabular}{lcccc}
\hline $\begin{array}{l}\text { Tamano } \\
\text { excuela }\end{array}$ & $\begin{array}{c}\text { Diferen } \\
\text { medias }\end{array}$ & $\begin{array}{c}\text { Coxiente } \\
\text { "t" }\end{array}$ & $\begin{array}{c}\text { Grados } \\
\text { libertad } \\
\text { (gl) }\end{array}$ & $\begin{array}{c}\text { Nivel de } \\
\text { signifi- } \\
\text { canciat (P) }\end{array}$ \\
\hline $\mathrm{T}-3$ & 0.5127 & 0.65 & 144 & 0.001 \\
$\mathrm{~T}-\mathrm{-1}$ & 0.7118 & 0.59 & 95 & 0.59 \\
$\mathrm{~T}-5$ & 1.6375 & 3.20 & 433 & 0.48 \\
TOTAL & 1.1431 & 2.81 & 676 & 0,033 \\
\hline
\end{tabular}

Los resultados obtenidos al aplicar la prueba " $t$ " para medias desiguales $(\mathrm{tc}=0,65)$ whlecen que no hay diferencia significativa in el rendimiento académico en Matemática -atre los estudiantes de escuelas que llevan el ?. $\mathrm{F}$. r respecto a los de NO P.I.E. en las socuekin de tamaño 3.

Pur =cuelas de tamaño 4, se observa auc antas nutras (PIE y NO PIE) provienen ce himina inuestra poblacional al obtener una $P=54$. on una t-test de igualdad

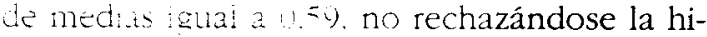
potesis nuta va que no existe diferencia a un nivel de contianza del $95 \%$. en el rendimiento acadenico un Matcmática en estudiantes de escuelas con el P.i.e. v los de NO P.I.E.

En las escuelas de tamano 5 , los dos grupos muestrales provienen de una misma poblacion al obtenerse una probabilidad de igualdad de varianza de $\mathrm{P}=0.479$. Puesto que la " $t$ " obtenida es de 3,20, se rechaza la hipótesis nula a un nivel de confianza del $95 \%$. Por tanto, sí existe diferencia significativa, obteniendo un mejor rendimiento académico en Matemática los estudiantes que no tienen Programa de Informática Educativa, que las escuelas con P.I.E.

En relación al grupo total de estudiantes, las dos muestras provienen de poblaciones diterentes al obtener una $P=0.033$ y una " $t$ " oba..... 2 2. I que indica que se acepta la mpos de invertagación a un nivel de conañza $\mathrm{Au}=$

Pas anto se conciuye que existe un and rencimiento académico en la asignatura se Matemática en la Dirección Regional de - itucación de san josé en estudiantes de quinic grado que no tienen en sus escuelas el Programa de Informática Educativa, que en aquellos estudiantes que sí llevan el P.I.E.

\section{Conclusiones}

Los resultados analizados anteriormente constituyen una respuesta a la interrogante que originó el estudio. Se puede concluir que:

Los estudiantes de escuelas de tamaño 5 presentan medias aritméticas más altas. que las escuelas de tamaños 3 y 4 . Lo que significa que los estudiantes de $\mathrm{T}-\overline{5}$ 
presentan puntajes más altos en la prueba de Matemática que los demás estudiantes.

En la muestra total el grupo NO P.I.E. (X $=16,43$ ) obtuvo una media aritmética mayor a la del P.I.E. ( $X=15,29)$. Presentando un mejor rendimiento académico en Matemática los estudiantes NO P.I.E.

Se logra determinar que la diferencia de medias aritméticas en los tres tamaños de escuelas y grupo total, es positivo y creciente conforme aumenta el tamaño de la muestra, estableciéndose un mayor rendimiento en los estudiantes que no participan del P.I.E.

Se logró determinar que en las escuelas de tamaños 3 y 4 no existe diferencia significativa a un nivel de confianza del $95 \%$ en rendimiento académico en la asignatura de Matemática a nivel de quinto grado entre estudiantes que participan del Programa de Informática Educativa con respecto a estudiantes de escuelas que no participan de éste.

En las escuelas de tamaño 5 . si existe diferencia significativa, a un nivel de confianza del $95 \%$ en el rendimiento académico en la asignatura de Matemática, presentando un valor significativamente mayor los del grupo NO P.I.E. que los estudiantes del P.I.E.

Con respecto al grupo total y tema central del estudio, se concluye que sí existe diferencia significativa en el rendimiento académico en Matemática entre estudiantes del NO P.I.E. y del P.I.E. Presentando un rendimiento significativamente mayor los estudiantes del NO P.I.E. que los estudiantes que les imparten lecciones del P.I.E.

Los resultados obtenidos evidencian compatibilidad con los de otros estudios similares a esta investigación.

\section{Bibliografia}

Azofaifa, Ana Isabel. (1978). Relación entre rendimiento académico, babilidad general y algunos aspectos de la enseñanza de la Matemática de estudiantes de un décimo año. Tesis. San José, Escuela de
Administración Educativa, Facultad de Educación, Universidad de Costa Rica.

Bigge, M. (1988). Teorias de aprendizaje para maestros. México, D.F., Editorial Trillas.

Blanco, Sandra y otros. (1981). Estudio del desarrollo de las nociones lógico-matemáticas segün Piaget y la facilidad de expresión ante las indicaciones orales y su relación con otras variables. Tesis. San José, Escuela de Formación Docente. Facultad de Educación, Universidad de Costa Rica.

Dobles, Cecilia y otros. (1995). Evaluación formativa. Programa de Informática Educativa (MEP-FOD). San José, Costa Rica, Fundación Omar Dengo.

Dobles, Cecilia y otros. (1994). Evaluación macroanalitica de contexto sobre una muestra de las escuelas que atiende. San José, Costa Rica, Fundación Omar Dengo.

Dobles, Cecilia y ZUÑIGA, Magaly. (1994). Significaciones en el contexto escolar $y$ actividad cognitiva. San José, Costa Rica.

Falbel, Aaron. (1993). Artículo "El Construccionismo". Traducido por Eleonora Badilla para el P.I.E. Fundación Omar Dengo.

Fonseca, Clotilde. (1991). Computadoras en la escuela pública costarricense. San José, Costa Rica, Editorial Gala, S.A.

Fonseca, Clotilde. (1988). La incorporación de la informática al sistema educativo y la experiencia de Costa Rica. Revista Praxis. No. 35-36.

Fonseca, Clotilde y SHAFFER, Marilyn. (1990). ¿Por qué Logo? Una respuesta para Costa Rica. San José, Costa Rica, Fundación Omar Dengo.

García, Jackeline. (1995). Implicaciones de los paradigmas en las conceptualizaciones pedagógicas. San José, Costa Rica, Fundación Omar Dengo. 
Gonzaléz Gairaud, Anny. (1994). El papel de las interacciones ante un objeto real. Coordinado por la Universidad de Río Grande del Sur, Laboratorio de Estudios Cognitivos.

Kerlinger, Fred. (1982). Investigación del comportamiento técnico y metodologías. México, D.F., Editorial Interamericana.

Nerici, Imideo. (1973). Hacia una didáctica general dinámica. México, D.F., Lapeluz.

Papert, Seymour. (1987). Desafio a la mente. Buenos Aires, Ediciones Galápagos.

Pardo Sáncez, Jorge. (1990). ¿Es la Matemática una disciplina que sólo mentes privilegiadas pueden aprender? Revista Praxis y Educación. No. 1.
Runyon y Haber (1992). Estadística para Ciencias Sociales. México, D.F., Editorial Addison Wesley.

Vargas, M., Salas, Z. y Víquez, S. (1993). Evaluación del Programa de Informática Educativa de I y II Ciclos. San José, Costa Rica, Editorial Publicaciones MEP.

Zúniga Céspedes, Magaly. (1995). ¿Epistología y/o ética? San José, Costa Rica, Fundación Omar Dengo.

Zúñiga García y otros. (1993). Impacto del Programa de Informática Educativa MEP-FOD en una escuela de zona mural. San José, Costa Rica, Fundación Omar Dengo. 